\title{
Spatial Distribution of Dislocations in Relation to a Substructure in High-Quality GaN Film
}

\author{
Mino Yang, ${ }^{1,2}$ Chong-Don Kim, ${ }^{3}$ Hee-Goo Kim, ${ }^{1}$ and Cheol-Woong Yang ${ }^{2, *}$ \\ ${ }^{1}$ Analytical Engineering Group, Samsung Advanced Institute of Technology (SAIT), Youngin, Gyeonggi-do 446-712, Korea \\ ${ }^{2}$ School of Advanced Materials Science \& Engineering, Sungkyunkwan University, Suwon, Gyeonggi-do 440-746, Korea \\ ${ }^{3}$ Analysis Lab, Samsung Corning Precision Materials, Gumi, Gyeongsangbuk-do 730-717, Korea
}

\begin{abstract}
The dislocation distribution of high-quality single-crystal gallium nitride (GaN) films grown by the hybrid vapor phase epitaxy was analyzed. This study examined the domain structure of GaN from the dislocation distribution on the macroscale by optical microscopy. The surface structure of GaN consisted of domains with microcolumns as the substructure. The inner domains contained a lower density of dislocations but a large number of these dislocations were observed along the domain boundaries. The existence of a domain boundary structure doubly increased the total dislocation density.
\end{abstract}

Key words: etch pit, dislocation, GaN, grain boundary, HVPE, TEM

\section{INTRODUCTION}

Gallium nitride (GaN)-based devices, which are highly defective unlike other III-V semiconductor, show singlecrystal quality in the optical performance. A dislocation density of $\sim 10^{9} / \mathrm{cm}^{2}$ in GaN-based light-emitting diodes (LEDs) does not lead to a pronounced decrease in lifetime (Nakamura et al., 1995). In wurtzite GaN, common threading screw and threading edge dislocations are electrically inactive (Elsner et al., 1997). Nevertheless, the demands for obtaining high-quality devices, such as laser diodes or boosting the output to produce high-power LEDs require a better understanding of dislocations in GaN.

In addition to the dislocation density, addressing its distribution is important because the GaN substrates used for optical devices are produced by film deposition methods. Practical quasi-single-crystalline $\mathrm{GaN}$ are obtained hetero-epitaxially on a single crystal, such as sapphire or $6 \mathrm{H}-\mathrm{SiC}$ through molecular organometallic vapor phase epitaxy or hybrid vapor phase epitaxy (HVPE; Amano et al., 1986; Detchprohm et al., 1992; Paisley \& Davis, 1993). Such GaN films are composed of microcolumns aligned to a certain orientation related to the hetero-epitaxial substrate. This makes them almost parallel, and small misorientations between the microcolumns are accommodated by dislocations leading to their coalescence (Qian et al., 1995; Ning et al., 1996). The majority of threading dislocations have been reported to be subsequently generated at the coalescence of the microcolumns (Wu et al., 1998). This suggests that these dislocations lay along the coalescence boundary of the microcolumn. Our study focused on the spatial distribution of dislocations in high-quality $\mathrm{GaN}$, and went further to confirm that the micrograins form clusters of high crystalline quality. This was achieved by a macroscopic

๑) MICROSCOPY SOCIETY OF AMERICA 2013

${ }^{\star}$ Corresponding author. E-mail: cwyang@skku.edu observation of the GaN surface with intentional oxidizing on dislocation sites.

\section{Materials and Methods}

Two low dislocation density GaN substrates were provided by Samsung Corning Precision Materials Ltd. Both were grown on a single-crystal (0001) $\alpha$-sapphire substrate to more than $200 \mu \mathrm{m}$ in thickness by a HVPE method under the same growth conditions except for the final cooling stage. The growth for such low dislocation density $\mathrm{GaN}$ in HVPE can be referred to Andre et al. (2007). One was grown normally by end (hereinafter referred to as "normal GaN"). The other was oxidized at the end of the growth stage. A very small amount of air was allowed to flow into the furnace at the cooling stage from the growth temperature $\left(\sim 900^{\circ} \mathrm{C}\right)$ to room temperature (hereinafter referred to as "oxidized GaN"). This was proposed to cause severe oxidation of the area where dislocations are located. Subsequently, a piece of oxidized GaN was placed in $\mathrm{P}_{2} \mathrm{O}_{5}$ at $190^{\circ} \mathrm{C}$ for $10 \mathrm{~min}$ to remove the oxide layer (hereinafter referred to as "etched GaN"). The effect was similar to that of the etching methods resulting in the formation of etch pits at the dislocation sites by the $\mathrm{SiH}_{4}$ treatment in the presence of $\mathrm{NH}_{3}$ (Oliver et al., 2006b). Oxidation at the high temperature applied in the present study produced large pits that were observable by optical microscopy.

Optical microscopy and transmission electron microscopy (TEM, Tecnai F20st, FEI) operating at $200 \mathrm{kV}$ equipped with a field-emission gun were used for the macroscopic and microscopic observations of the microstructure, respectively. The elemental composition was measured by energydispersive spectrometry (EDS). Plan-view and cross-sectional TEM specimens were prepared by a conventional technique involving mechanical polishing followed by ion beam milling. To characterize the dislocation, the GaN samples were examined by WBDF with diffraction spots $\mathbf{g}_{1}=002$ and 

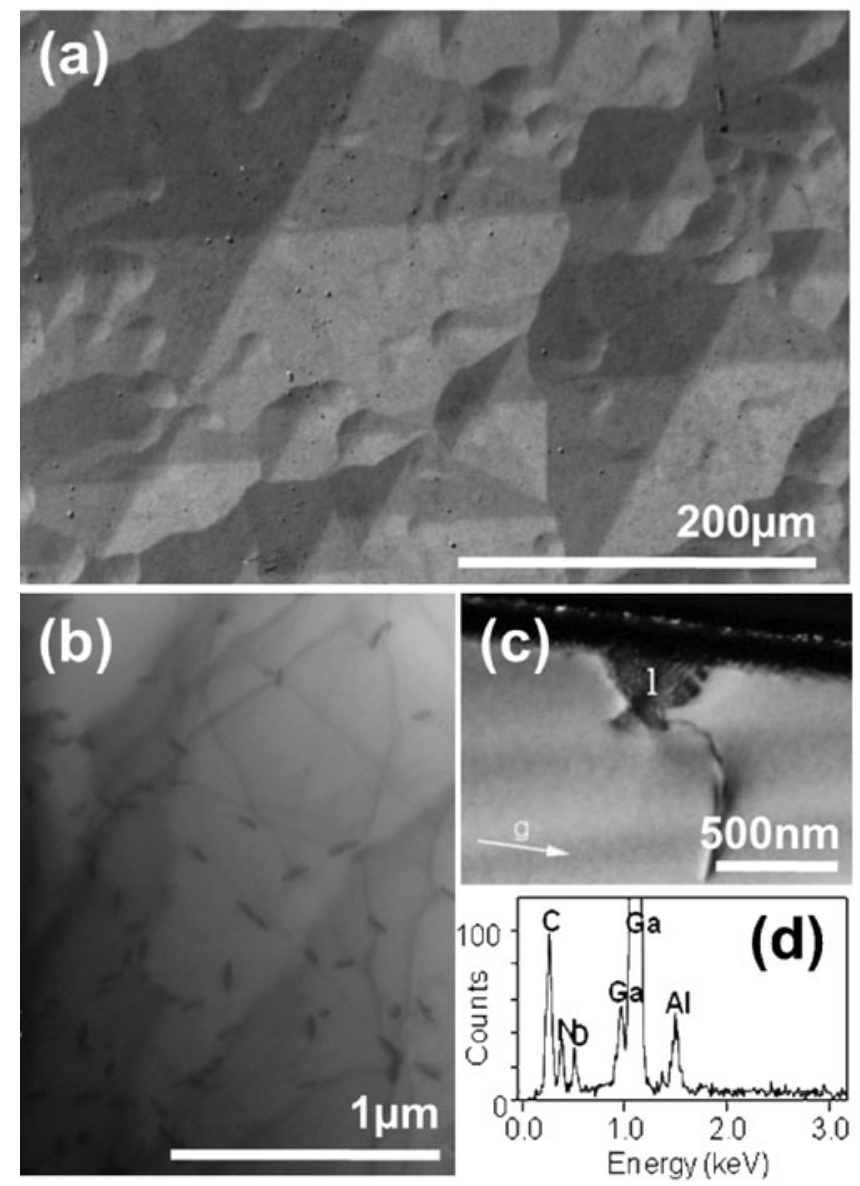

Figure 1. a: The surface morphology of "etched GaN" observed by optical microscope. b: Plan view transmission electron microscopy (TEM) image of the "oxidized GaN." The density and distribution of the oxides are similar to those of the surface dislocations of the "normal GaN." c: Cross-sectional TEM image of the oxide filled pit that corresponds to dark features in (b). d: Energy-dispersive spectrometry spectrum at the area marked by " 1 " in (c) indicating that the area consists of gallium oxide.

$\mathbf{g}_{2}=1-10$ under two-beam conditions using a $3 \mathbf{g}$ diffraction spot, following the invisible criterion of $\mathbf{g} \cdot \mathbf{b}=0$, where $\mathbf{g}$ is the reciprocal lattice vector and $\mathbf{b}$ is Burger's vector (Williams \& Carter, 1996). The dislocation density was measured on plan-view specimens, which were observed with an $18^{\circ}$ specimen tilt from $\mathbf{g}=001$ to the direction of $\mathbf{g}=$ 110 , allowing for observation of the dislocation characteristics (Follstaedt et al., 2003).

\section{Results And Discussion}

The morphology of the GaN surface was roughened by the existence of domains. In optical microscopy observations, the "normal," "oxidized," and "etched GaN" showed similar surface structures that consisted of a pyramidal-shaped domain (Domain) in the radius ranging from the tens to mostly more than hundreds of micrometers as shown in Figure 1a. According to the results of the cross-sectional (see Fig. 1c) and plane view (see Fig. 1b) TEM observations, pits

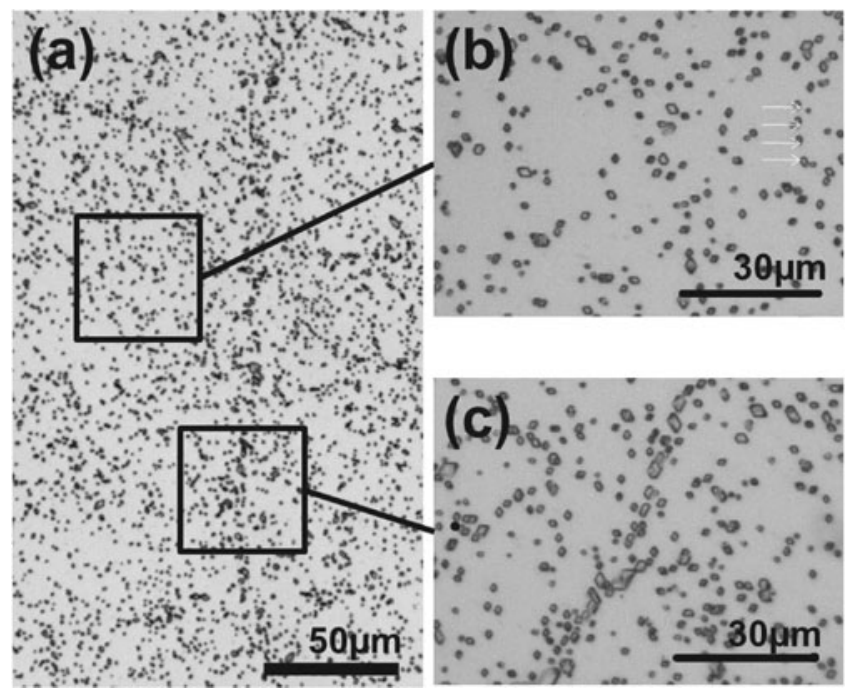

Figure 2. Optical micrographs of the "etched GaN." a: Highdensity dislocation array along the domain boundary. b,c: Enlarged inner domain area and the area containing domain boundary, respectively. The pit density (equal to dislocation density) was measured to be $4.2 \times 10^{6} / \mathrm{cm}^{2}$ in the inner domain area that is comparable to the total dislocation density of $9.9 \times 10^{6} / \mathrm{cm}^{2}$.

filled with gallium oxide (see EDS result in Fig. 1d) were observed on the surface of the "oxidized GaN" with a spatial density of $8.9 \times 10^{6} / \mathrm{cm}^{2}$. They were then removed by phosphoric acid, which left pits on the surface (the "etched GaN"). Dislocations were observed below every pit with the types of edge, mixed, and rarely screw using WBDF. The pit density (number per area) in the "etched GaN" was $9.9 \times$ $10^{6} / \mathrm{cm}^{2}$ according to the recorded optical micrographs. The dislocation density measured on the surface of normal GaN by TEM was $9.1 \times 10^{6} / \mathrm{cm}^{2}$. A slightly lower dislocation density than the pit density was observed, and the difference is within the known TEM measurement error of $10 \%$ (Follstaedt et al., 2003). Briefly, the pits are considered to be the sites of dislocation, making a visible dislocation distribution on the optical microscopic scale.

In contrast to the $\mathrm{GaN}$ used in the published reports on the coalescence boundary, the present $\mathrm{GaN}$ had a low defect density $\left(\sim 10^{7} / \mathrm{cm}^{2}\right)$ and showed an as-deposited domain structure with typical hexagonal stripes on the surface. The stripes will be the tangential lines between the plane $\{112\}$ or $\{110\}$ with respect to the growth conditions (Hiramatsu et al., 1999; their characterization will not be reported here). The straight hexagonal stripes imply that the individual domains are single-crystals despite the significant number of dislocations accommodating the near-parallel misorientation of the constituent microcolumns. In "etched GaN," pits are distributed discretely over the inner domain area (Figs. 2a, 2b). The mean inter-distance of the dislocation measured in the inner domain area was $\sim 4.9 \mu \mathrm{m}$. From the assumption that a domain maintains a rather high quality of local single crystalline structure, the randomly oriented constituent microcolumns are believed to be accommodated by the inconsistent sign of the dislocation. As the present $\mathrm{GaN}$ has a low disloca- 
tion density, applying the equation, $\tan (\Delta \theta) \approx \Delta \theta=\mathbf{b} / D$, to the approximated misorientation between the microcolumn gives almost $0^{\circ}$, where $\Delta \theta$ is the angle between the microcolumn in $c$-axial rotation, $D$ is the dislocation's inter-distance, and $\mathbf{b}$ is Burger's vector corresponding to the dislocation type (Ning et al., 1996). The inter-distance of the dislocation will be better for representing the degree of misoriention.

Misorientation became larger at the domain boundary. Boundaries crowded by high-density dislocations were observed in the present GaN, as shown in Figure 2c. These boundaries are distinguished from the dislocation distribution of the inner area, and are rather close to the low-angle grain boundary which is an array of line defects superimposed on a singular configuration (Pond, 1995). By comparing the dislocation density of the inner domain $(4.2 \times$ $10^{6} / \mathrm{cm}^{2}$ ), the existence of domain boundaries increased the entire dislocation density by almost twice $\left(9.1 \times 10^{6} / \mathrm{cm}^{2}\right)$. The mean inter-distance of the dislocation was measured $\sim 2.7 \mu \mathrm{m}$ along the boundary.

Even though the origin is unclear, dislocation arrays often observed in GaN films have been regarded as coalescence boundaries (Qian et al., 1995; Ning et al., 1996). Arrays of dislocations were used to form a low-angle grain boundary. However, recent reports on the characterization of the coalescence boundary in the GaN film conversely suggest that they are not due to the coalescence. The alignment of dislocations was adopted in the system for the purpose of relieving the internal strain, which was derived from the result of continuum elastic calculation by Gmeinwieser and Schwarz (2007). This was confirmed by atomic force microscopy of the partially coalesced GaN, where no dislocations were generated at the region where two micrograins coalesced (Oliver et al., 2006a). The arrays of dislocations were observed in the inner grain and laid across the apparent boundary. In such cases, the initial misorientation between the grains should be accommodated by a slight distortion of the lattice (Moram et al., 2009). The dislocation array examined in previous reports was limited from several to 20 individual dislocations.

The similarly distributed (equally spaced with small number) dislocation arrays were found in the inner domain area of the present specimen, as indicated by arrows in Figure 2b. Previous reports (Oliver et al., 2006a; Gmeinwieser \& Schwarz, 2007; Moram et al., 2009) appear to describe the dislocation distribution of the inner domain, or the difference from ours would be derived from different growth conditions with respect to the growth anisotropy realized by the manufacturers (Hiramatsu et al., 1999). In contrast, domain boundaries with more than one hundred dislocations were found frequently in the present GaN. These dislocations would be a mixture of them originating from the inner domain and the misorientation between domains. Removing the contribution of dislocations to the inner domain gives an $\sim 6.04 \mu \mathrm{m}$ inter-dislocation distance as a very small misorientation between domains in the case of Figure 2a. However, the real misorientation will deviate largely because it is not certain that the dislocation has a consistent sign of the Burgers vector like a typical low-angle grain boundary.

Consequently, the clustering of microcolumns results in a domain structure. Even if it is unclear whether the boundaries originated from the coalescence between domains or it was adopted for lowering energy by rearranging dislocations during growth, the clustering of microcolumn seems to produce locally high-quality GaN crystal. In this sense, the domain boundary is comparable to the wing boundary appearing in the lateral overgrowth method (Romanov et al., 2003).

\section{SUMMARY}

This study examined the surface of high-quality GaN films grown by HVPE, and a domain structure consisting of microcolumns was found in the substructure. The pile-up of dislocations along the domain boundary highly increased the entire dislocation density. Coupled to the device, the domain boundary will provide high density of dislocations resulting in performance failure. This suggests that the domain boundary needs to be avoided because it provides a dominant failure source when it runs through the active area in devices. Information on the domain boundary structure can be used to qualify the GaN substrates in relation to the dislocation density.

\section{ACKNOWLEDGMENTS}

This work was supported by the National Research Foundation of Korea (NRF) grants funded by the Korea government (MEST; No. 2011-0017257, No. 2011-0030803, and No. 2011-0019984). The authors gratefully appreciate technical support from the cooperative center for research facilities (CCRF) at Sungkyunkwan University.

\section{RefERENCES}

Amano, H., Sawaki, N., Akasaki, I. \& Toyoda, Y. (1986). Metalorganic vapor phase epitaxial growth of a high quality GaN film using an AlN buffer layer. Appl Phys Lett 48, 353-355.

Andre, Y., Trassoudaine, A., Tourret, J., Cadoret, R., Gil, E., Castelluci, D., Aoude, O. \& Disseix, P. (2007). Low dislocation density high-quality thick hydride vapour phase epitaxy (HVPE) GaN layers. J Crystal Growth 306, 86-93.

Detchprohm, T., Hiramatsu, K., Amano, H. \& Akasaki, I. (1992). Hydride vapor phase epitaxial growth of a high quality GaN film using a ZnO buffer layer. Appl Phys Lett 61, 2688-2690.

Elsner, J., Jones, R., Sitch, P.K., Porezag, V.D., Elstner, M., Frauenheim, T., Heggie, M.I., Öberg, S. \& Briddon, P.R. (1997). Theory of threading edge and screw dislocations in GaN. Phys Rev Lett 79, 3672-3675.

Follstaedt, D.M., Missert, N.A., Koleske, D.D., Mitchell, C.C. \& Cross, K.C. (2003). Plan-view image contrast of dislocations in GaN. Appl Phys Lett 83, 4797-4799.

Gmeinwieser, N. \& Schwarz, U.T. (2007). Pattern formation and directional and spatial ordering of edge dislocations in bulk GaN: Microphotoluminescence spectra and continuum elastic calculations. Phys Rev B 75, 245213. 
Hiramatsu, K., Nishiyama, K., Motogaito, A., Miyake, H., Iyechika, Y. \& MaedA, T. (1999). Recent progress in selective area growth and epitaxial lateral overgrowth of III-nitrides: Effects of reactor pressure in MOVPE growth. Phys Stat Sol (a) 176, 535-543.

Moram, M.A., Oliver, R.A., Kappers, M.J. \& Humphreys, C.J. (2009). The spatial distribution of threading dislocations in gallium nitride films. Adv Mater 21, 3941-3944.

Nakamura, S., Senoh, M., Iwasa, N., Nagahama, S., Yamada, T. \& MukaI, T. (1995). Superbright green InGaN single-quantumwell-structure light-emitting diodes. Jpn J Appl Phys 34(10B), L1332-L1335.

Ning, X.J., Chien, F.R., Pirouz, P., Yang, J.W. \& Asif Khan, M. (1996). Growth defects in GaN films on sapphire: The probable origin of threading dislocations. J Mater Res 11, 580-592.

Oliver, R.A., Kappers, M.J. \& Humphreys, C.J. (2006a). Insights into the origin of threading dislocations in $\mathrm{GaN} / \mathrm{Al}_{2} \mathrm{O}_{3}$ from atomic force microscopy. Appl Phys Lett 89, 011914.

Oliver, R.A., Kappers, M.J., Sumner, J., Datta, R. \& HumPhreys, C.J. (2006b). Highlighting threading dislocations in
MOVPE-grown GaN using an in situ treatment with $\mathrm{SiH}_{4}$ and $\mathrm{NH}_{3}$. J Crystal Growth 289, 506-514.

Paisley, M.J. \& Davis, R.F. (1993). Molecular beam epitaxy of nitride thin films. J Crystal Growth 127, 136-142.

Pond, R.C. (1995). On the characterization of interfacial defects using high resolution electron microscopy. Interface Sci 2, 299-310.

Qian, W., Skowronski, M., de Graef, M., Doverspike, K., Rowland, L.B. \& GASKILL, D.K. (1995). Microstructural characterization of $\alpha$-GaN films grown on sapphire by organometallic vapor phase epitaxy. Appl Phys Lett 66, 1252-1254.

Romanov, A.E., Fini, P. \& Speck, J.S. (2003). Modeling the extended defect evolution in lateral epitaxial overgrowth of $\mathrm{GaN}$ : Subgrain stability. J Appl Phys 93, 106-114.

Williams, D.B. \& Carter, C.B. (1996). Transmission Electron Microscopy. New York: Plenum.

Wu, X.H., Fini, P., Tarsa, E.J., Heying, B., Keller, S., Mishra, U.K., Den BAARs, S.P. \& Speck, J.S. (1998). Dislocation generation in GaN heteroepitaxy. J Crystal Growth 189/190, 231-243. 